\title{
Polymorphisms of IKZF3 Gene and Autoimmune Thyroid Diseases: Associated with Graves' Disease but Not with Hashimoto's Thyroiditis
}

\author{
Ling Lia Xiaolian Ding ${ }^{b} \quad$ Xuan Wang ${ }^{a}$ Qiuming Yao ${ }^{a}$ Xiaoqing Shao \\ Xiaofei An ${ }^{c} \quad N_{\text {Yan }}^{a}$ Yanfei Jiang ${ }^{a}$ Wen Wang ${ }^{a}$ Liangfeng Shia Qiu Qin ${ }^{a}$ \\ Ronghua Song ${ }^{a}$ Jin-an Zhang ${ }^{a}$ Peilong Sun ${ }^{d}$
}

aDepartment of Endocrinology, Jinshan Hospital of Fudan University, Shanghai, 'bepartment of Nephrology and Endocrinology, Weinan Central Hospital of Shaanxi Province, Weinan, 'Department of Endocrinology, The Affiliated Hospital of Nanjing University of Chinese Medicine, Nanjing, dDepartment of General Surgery, Jinshan Hospital of Fudan University, Shanghai, China

\section{Key Words}

Ikaros family of zinc finger3 (IKZF3) - Single nucleotide polymorphism (SNP) - Autoimmune thyroid disease (AITD) • Graves' disease (GD) • Hashimoto's thyroiditis (HT)

\begin{abstract}
Background/Aims: The IKZF3 gene encodes a zinc-finger protein that plays an important role in the proliferation and differentiation of $B$ lymphocytes. Autoimmune thyroid diseases (AITDs), mainly include Graves' disease (GD) and Hashimoto's thyroiditis (HT), are probably caused by the aberrant proliferation of B cells. The objective of this study was to explore the association between IKZF3 polymorphisms and AITDs. Methods: We examined 915 AITD patients (604 GD and $311 \mathrm{HT}$ ) and 814 healthy controls. IKZF3 variants (rs2941522, rs907091, rs1453559, rs12150079 and rs2872507) were tested by PCR-ligase detection reaction. Results: It was manifested that that the minor alleles of the five loci increased susceptibility to GD $(p<0.05$ for $r s 2941522$, and $p<0.01$ for rs907091, rs1453559, rs12150079 and rs2872507) but in HT patients, these loci showed no significant difference compared with controls. Similarly, the genotype distributions of GD patients manifested obvious differences in all these loci compared with the control group, whereas no statistical differences were observed between HT patients and controls. Furthermore, bioinformatics tools were used to analyze rs1453559, rs12150079 and rs907091. These variants were believed to be the transcription regulator. Conclusion: It is the first time we reported the association between the IKZF3 polymorphisms and GD, indicating that IKZF3 gene tends to bean important risk factor for the development of GD.

Jin-an Zhang

and Peilong Sun
Department of Endocrinology, Jinshan Hospital of Fudan University, Shanghai,

Department of General Surgery, Jinshan Hospital of Fudan University, Shanghai (China) E-Mail zhangjinan@hotmail.com, peilong@sh163.net

\section{KARGER}




\section{Introduction}

Autoimmune thyroid diseases (AITDs), including Graves' disease (GD) and Hashimoto's thyroiditis (HT), are the common organ-specific autoimmune disorders affecting the thyroid. Its etiology appears to be the interactions between genetic and environmental factors. GD and HT are characterized by immunocytes' imbalance and autoantibodies' production [1]. The HLA-DR3 gene was identified as a susceptible region for AITD [2]. In addition, several types of non-HLA genes have been reported, including immunoregulatory genes like CD40, CTLA-4, PTPN22 and thyroid-specific genes, such as variants for thyroid stimulating hormone receptor (TSHR) and thyroglobulin (TG). In recent years, with the achievement in Human Genome Project and the genome-wide association (GWA) studies, a number of new loci have been identified as the risk factors of Graves' disease [3]. In this study, we examined the Ikaros family of zinc finger3 (IKZF3) gene, novel susceptible variants, in AITDs patients.

The IKZF3 gene is located on human chromosome 17q12-q21.1, consisting of 9 exons and spanning a region of $104 \mathrm{~kb}$. This gene encodes a zinc-finger protein, Aiolos, which is a member of the Ikaros family. The Ikaros family includes 3 members - Ikaros, Aiolos and Helios. Each is hematopoietic-specific transcription factor that regulates the development of lymphocytes. The product of IKZF3 gene is expressed highly in mature peripheral B cells, and also detected in T and B cell precursors [4]. The IKZF3 not only participates in chromatin remodeling and histone deacetylation, but also is an important transcription factor inhibiting B cell proliferation and differentiation [5, 6]. The IKZF3-deficient mice exhibited an activated cell surface phenotype, leading to the formation of germinal centers and overproduction of serum IgG and IgE [7]. As AITDs are prone to be aroused by the excessive differentiation of plasma cells and overproduction of autoantibodies, the IKZF3 might be a susceptibility gene for GD or HT.

Many recent studies have suggested several SNPs of IKZF3 gene have significant associations with systemiclupus erythematosus (SLE), a representative systemic autoimmune disease $[8,9]$. It is also demonstrated that the IKZF3-mutant mice would developed into the SLE-like phenotypes [10]. Besides SLE, several IKZF3 SNPs are also associated with rheumatoid arthritis (RA) [11], asthma [12-14] and primary biliary cirrhosis (PBC) [15], indicating its comprehensive involvement with autoimmune or immunological diseases. Up to now, few researches have investigated the relationship between IKZF3 polymorphisms and AITDs. Therefore, we performed a case-control study to explore the genetic association between IKZF3 and AITDs.

\section{Materials and Methods}

\section{Patients and Controls}

In our study, we investigated a total of 1729 subjects, including 915 AITD patients (604 GD patients and 311 HT patients) and 814 healthy controls. Inclusion criteria: Individuals were diagnosed with GD by clinical and laboratory confirmed hyperthyroidism, added with diffuse goiter and positive anti-thyroid stimulating hormone receptor antibody (TRAb). HT was diagnosed by enlarged thyroid, either positive anti-thyroid peroxidase antibody (TPO-Ab) or positive anti-thyroglobulin antibody (Tg-Ab), with/without hypothyroidism. Exclusion criteria: Healthy controls were screened for the absence of goiter and negative TPO-Ab. Controls suffered from or with family history for any immune-related diseases were excluded. Patients with other autoimmune diseases except for AITDs were also excluded, such as rheumatoid arthritis and SLE. All of the subjects were Chinese Han ethnicity and unrelated with each other. Patients with AITDs and controls were recruited separately from the department of Endocrinology and the Health Care Center, Jinshan Hospital of Fudan University in Shanghai. This study received the IRB (Institutional Reviewer Board) approval from Jinshan Hospital of Fudan University. Informed consents were obtained from all participants (included AITD patients and controls). Demographic characteristics of all the participantsare presented in Table 1.

\section{KARGER}




\section{Cellular Physiology Cell Physiol Biochem 2018;45:1787-1796 \\ \begin{tabular}{l|l|l}
\hline DOI: 10.1159/000487870 & $\begin{array}{l}\text { C } 2018 \text { The Author(s). Published by S. Karger AG, Basel } \\
\text { www.karger.com/cpb }\end{array}$ \\
\hline
\end{tabular}}

Li et al.: IKZF3 Polymorphisms with GD

\section{DNA extraction and SNP genotyping}

We collected peripheral blood from all subjects. Genomic DNA was extracted by RelaxGene Blood DNA System (Tiangen Biotech, Beijing, China). IKZF3 SNPs were genotyped by PCR-ligase detection reaction (PCR-LDR) platform. IKZF3 SNPs were selected from the Hapmap CHB database by Haploview software 4.2 using LD-based tagSNP selection by LDSelect. We searched the IKZF3 chromosomal region and its upper/lower sitesto capture the 5' and 3' untranslated regions (UTR). All the SNPs were selected according to the following criteria: minor allele frequency (MAF) $>0.1$, Hardy-Weinberg equilibrium (HWE) $>0.05$, and logarithm of odds (LOD) > 3.0. Information of selected SNPs was shown in Table 2. The presumed functions of these variants were extracted from the database of Ensembl genome browser (www.ensembl.org). Rs1453559 is located within the 5'- untranslated region (UTR), and rs907091 within the 3'UTR of IKZF3. Rs2941522 is a downstream variant located at the 3' of IKZF3, and two SNPs of rs12150079 and rs2872507 at the 5' flanking region of the gene. The five SNPs are in one linkage disequilibrium block. A strong linkage disequilibrium (LD) was exhibited in these 5 SNPs $\left(D^{\prime} \geq 0.9\right.$ for each SNP combination). All the SNPs were located within the same haplotype, representing a single signal for association. Specific primers for these SNPs are shown in Table 3.

\section{Statistical analysis}

Clinical data were expressed as $\mathrm{M} \pm \mathrm{SD}$ (mean \pm standard deviation). HWE and LD were analyzed by Haploview software 4.2. Statistical analysis was performed using SPSS 21.0. SNP frequencies between the patients and the controls were compared by Pearson chi-square test or Fisher's exact test. Odds ratios $(O R)$, with the $95 \%$ confidence intervals $(95 \%$ $C I$ ) were calculated to express the risk of acquiring AITDs. $\mathrm{p}<0.05$ was considered to be statistically significant.

\section{Power Calculation}

The allele frequency of the selected SNPs was from 0.186 to 0.443 , which is proper for power calculation. The $604 \mathrm{GD}$ patients and 814 controls provided a power of $81.3 \%$ with an $O R=1.27$ at $p=0.05$ significance level. The power of the data was evaluated by the Power and Sample Size Calculation Software (http://biostat.mc.vanderbilt.edu/twiki/bin/ view/Main/Power-SampleSize).

\section{Functional prediction}

Potential functions of SNPs were extracted from SNPinfo (https://snpinfo.niehs.nih.gov/snpinfo/ snpfunc.html). We also used bioinformatics databases such as Promo (http://alggen.lsi.upc.es/cgi-bin/ promo_v3/promo/promoinit.cgi?dirDB=TF_8.3) to analyze transcription factor binding sites (TFBS). As
Table 1. Clinical statistics of AITD patients and controls

\begin{tabular}{lccc}
\hline & GD $(\%)$ & HT(\%) & Control(\%) \\
\hline Number & 604 & 311 & 814 \\
Gender & & & \\
Male & $189(31.29)$ & $39(12.54)$ & $291(35.75)$ \\
Female & $415(68.71)$ & $272(87.46)$ & $523(64.25)$ \\
Age & $37.20 \pm 14.69$ & $35.07 \pm 14.04$ & $38.73 \pm 9.05$ \\
Onset of age & $33.90 \pm 14.81$ & $33.51 \pm 14.40$ & \\
Goiter & & & \\
None & $110(18.21)$ & $42(13.50)$ & \\
I degree & $103(17.05)$ & $52(16.72)$ & \\
II degree & $315(52.15)$ & $196(63.02)$ & \\
III degree & $76(12.58)$ & $21(6.75)$ & \\
Ophthalmopathy & & & \\
(+) & $108(17.88)$ & $3(0.96)$ & \\
$(-)$ & $496(82.12)$ & $308(99.04)$ & \\
Thyroid antibodies & & & \\
TPO-Ab & $241(39.90)$ & $223(71.70)$ & \\
Tg-Ab & $322(53.31)$ & $199(63.99)$ & \\
Family history & & & \\
(+) & $126(20.86)$ & $63(20.26)$ & \\
$(-)$ & $478(79.14)$ & $248(79.74)$ & \\
Adolescent (<18) & $56(9.27)$ & $19(6.11)$ & $64(7.86)$ \\
Smoking & & & \\
Male & $98(51.85)$ & $12(30.76)$ & $136(46.73)$ \\
Female & $6(1.45)$ & $1(0.37)$ & $7(1.39)$ \\
Hypothyroidism & & $96(30.87)$ & \\
\hline
\end{tabular}

Table 2. Information of chosen SNPs. A strong LD was exhibited in these 5 SNPs ( $D^{\prime} \geq 0.9$ for each SNP combination). Chr, Chromosome; MAF, Minor Allele Frequency; HWE, Hardy-Weinberg Equilibrium

\begin{tabular}{lcccccc}
\hline \multirow{2}{*}{ SNP } & \multirow{2}{*}{ Base change } & Gene name & Chr & \multicolumn{2}{c}{ MAF } & Database \\
& Subjects & HWE \\
\hline rs2941522 & $\mathrm{A}>\mathrm{G}$ & IKZF3 (3' flank) & 17 & 0.443 & 0.313 & 0.847 \\
rs907091 & T>C & IKZF3 & 17 & 0.470 & 0.314 & 0.734 \\
rs1453559 & $\mathrm{A}>\mathrm{G}$ & IKZF3 & 17 & 0.457 & 0.279 & 0.784 \\
rs12150079 & $\mathrm{G}>\mathrm{A}$ & IKZF3 (5' flank) / ZPBP2 & 17 & 0.186 & 0.210 & 0.543 \\
rs2872507 & $\mathrm{G}>\mathrm{A}$ & IKZF3 (5' flank) & 17 & 0.326 & 0.277 & 0.624 \\
\hline
\end{tabular}

Table 3.The primer sequences of chosen SNPs at IKZF3gene region

\begin{tabular}{lcc}
\hline SNP & Primer & Sequences(5'-3') \\
\hline rs2941522 & Forward & GTGGGAACCTCGAGAGACAG \\
& Reverse & GTCGCGGCTCTTTGAACTC \\
rs907091 & Forward & TCTTAACCCTTCAGCCTGTGA \\
& Reverse & CAGGGACTTGAATTTTATGGAA \\
rs1453559 & Forward & TCCTTGGGTTTGACATTCG \\
& Reverse & AAAGACCCCGGAGAAAAGAA \\
rs12150079 & Forward & GGCTCAGCTCAAATGTCCTT \\
& Reverse & CAGAACGATGGTATGCTTGG \\
& Forward & ACATCATGTTGACACTTAGAAAAGTT \\
rs2872507 & Reverse & TAGCTCTATGCCAGAGTAAAATTG \\
\hline
\end{tabular}


for potential miRNA target site prediction, data were accessed from mirnada (http://www.microrna.org/ microrna/home.do).

\section{Results}

\section{Association of IKZF3 Polymorphism with GD and HT}

Genotype distributions for SNPrs2941522, rs907091, rs1453559, rs12150079 and rs2872507 were in the range of predicted HWE, no matter in the patient group or the control group. Allele and genotype frequencies of the five SNPs of patients and controls were listed in Table 4. Each of the five SNP was associated with AITDs $(\mathrm{p}<0.05)$ in allele frequency analysis, and the minor (variant) alleles of all the 5 SNPs were risk alleles, which probably induced individuals to AITDs. Genotyping analysis showed that four of five SNPs exhibited different frequencies between AITD patients and controls. Besides, as shown in Table 5, more individuals in AITD group carried mutant homozygotes than in the control group at the loci of rs907091, rs1453559, rs12150079 and rs2872507.

When GD and HT groups were analyzed separately, we noticed the allele distributions of rs2941522, rs907091, rs1453559, rs12150079 and rs2872507 showed obvious differences between GD individuals and controls $(\mathrm{p}=0.020, \mathrm{OR}=1.21 ; \mathrm{p}=0.006, \mathrm{OR}=1.25 ; \mathrm{p}=0.007$, $\mathrm{OR}=1.25 ; \mathrm{p}=0.006, \mathrm{OR}=1.29 ; \mathrm{p}=0.004, \mathrm{OR}=1.27$, respectively). Similarly, the mutant

Table 4. Allele and genotype distribution of the 5 SNPs in AITD patients and controls

\begin{tabular}{|c|c|c|c|c|c|c|c|}
\hline \multirow{2}{*}{ SNP ID } & \multicolumn{4}{|c|}{ Allele/genotype frequency n (\%) } & \multicolumn{3}{|c|}{$\mathrm{P}$ value $[\mathrm{OR}(95 \% \mathrm{CI})]$} \\
\hline & Control & AITD & $\mathrm{GD}$ & HT & AITDs vs. C & GD vs. C & HT vs. C \\
\hline \multicolumn{8}{|c|}{ rs2941522 } \\
\hline G & $479(29.42)$ & $605(33.06)$ & $405(33.53)$ & $200(32.15)$ & \multirow{3}{*}{$\begin{array}{c}0.021[1.19(1.03-1.37)] \\
\text { reference }\end{array}$} & \multirow{2}{*}{$\begin{array}{c}0.020[1.21(1.03-1.42)] \\
\text { reference }\end{array}$} & \multirow{2}{*}{$\begin{array}{c}0.207[1.14(0.93-1.39)] \\
\text { reference }\end{array}$} \\
\hline A & $1149(70.58)$ & $1225(66.94)$ & $803(66.47)$ & $422(67.85)$ & & & \\
\hline GG & $69(8.48)$ & $103(11.26)$ & $75(12.42)$ & $28(9.00)$ & & \multirow{3}{*}{0.036} & \multirow{3}{*}{0.329} \\
\hline AG & $341(41.89)$ & $399(43.61)$ & $255(42.22)$ & $144(46.30)$ & \multirow{2}{*}{0.064} & & \\
\hline AA & $404(49.63)$ & $413(45.13)$ & $274(45.36)$ & $139(44.69)$ & & & \\
\hline \multicolumn{8}{|c|}{ rs907091 } \\
\hline C & $474(29.12)$ & $609(33.27)$ & $410(33.94)$ & 199(31.99) & \multirow{3}{*}{$\begin{array}{c}0.008[1.21(1.05-1.40)] \\
\text { reference }\end{array}$} & \multirow{3}{*}{$\begin{array}{c}0.006[1.25(1.07-1.47)] \\
\text { reference }\end{array}$} & \multirow{3}{*}{$\begin{array}{c}0.182[1.15(0.94-1.39)] \\
\text { reference }\end{array}$} \\
\hline $\mathrm{T}$ & $1154(70.88)$ & $1221(66.73)$ & $798(66.06)$ & $423(68.01)$ & & & \\
\hline $\mathrm{CC}$ & $65(7.98)$ & $108(11.80)$ & $79(13.07)$ & $29(9.32)$ & & & \\
\hline CT & $344(42.26)$ & $393(42.95)$ & $252(41.72)$ & $141(45.33)$ & \multirow{3}{*}{0.017} & \multirow{3}{*}{0.006} & \multirow{2}{*}{0.390} \\
\hline TT & $405(49.75)$ & $414(45.24)$ & $273(45.19)$ & $141(45.33)$ & & & \\
\hline \multicolumn{6}{|c|}{ rs1453559 } & & \\
\hline G & $423(25.98)$ & $543(29.67)$ & $369(30.55)$ & $174(27.97)$ & \multirow{3}{*}{$\begin{array}{c}0.016[1.20(1.04-1.39)] \\
\text { reference }\end{array}$} & \multirow{3}{*}{$\begin{array}{c}0.007[1.25(1.06-1.48)] \\
\text { reference }\end{array}$} & \multirow{3}{*}{$\begin{array}{l}0.339[1.11(0.90-1.36)] \\
\text { reference }\end{array}$} \\
\hline A & $1205(74.02)$ & $1287(70.33)$ & $839(69.45)$ & $448(72.03)$ & & & \\
\hline GG & $48(5.89)$ & $84(9.18)$ & $62(10.26)$ & $22(7.07)$ & & & \\
\hline $\mathrm{AG}$ & $327(40.17)$ & $375(40.98)$ & $245(40.46)$ & $130(41.80)$ & \multirow{3}{*}{0.023} & \multirow{3}{*}{0.007} & \multirow{2}{*}{0.612} \\
\hline AA & $439(53.93)$ & $456(49.83)$ & $297(49.17)$ & $159(51.12)$ & & & \\
\hline \multicolumn{6}{|c|}{ rs12150079 } & & \\
\hline A & $312(19.17)$ & $415(22.68)$ & $283(23.43)$ & $132(21.22)$ & \multirow{3}{*}{$\begin{array}{c}0.011[1.24(1.05-1.46)] \\
\text { reference }\end{array}$} & \multirow{3}{*}{$\begin{array}{c}0.006[1.29(1.08-1.55)] \\
\text { reference }\end{array}$} & \multirow{3}{*}{$\begin{array}{c}0.273[1.14(0.90-1.43)] \\
\text { reference }\end{array}$} \\
\hline G & $1316(80.83)$ & $1415(77.32)$ & $925(76.57)$ & $490(78.78)$ & & & \\
\hline AA & $29(3.56)$ & $52(5.68)$ & $36(5.96)$ & $16(5.14)$ & & & \\
\hline $\mathrm{AG}$ & $254(31.20)$ & $311(33.99)$ & $211(34.93)$ & $100(32.15)$ & \multirow{2}{*}{0.033} & 0019 & 0428 \\
\hline GG & $531(65.23)$ & $552(60.33)$ & $357(59.11)$ & $195(62.70)$ & & 0.019 & 0.428 \\
\hline rs2872 & & & & & & & \\
\hline A & $417(25.61)$ & $541(29.56)$ & $368(30.46)$ & $173(27.81)$ & $0.010[1.22(1.05-1.42)]$ & $0.004[1.27(1.08-1.50)]$ & $0.289[1.12(0.91-1.38)]$ \\
\hline G & $1211(74.39)$ & $1289(70.44)$ & $840(69.54)$ & $449(72.19)$ & reference & reference & reference \\
\hline AA & $46(5.65)$ & $82(8.96)$ & $61(10.09)$ & $21(6.75)$ & & & \\
\hline AG & $325(39.92)$ & $377(41.20)$ & $246(40.72)$ & $131(42.12)$ & & & \\
\hline GG & $443(54.42)$ & $456(49.83)$ & $297(49.17)$ & $159(51.12)$ & 0.016 & 0.004 & 0.553 \\
\hline
\end{tabular}

Table 5. Comparisons of genotype frequencies in AITD patients and controls

\begin{tabular}{|c|c|c|c|c|c|c|c|}
\hline \multirow{2}{*}{ SNP ID } & \multicolumn{4}{|c|}{ Allele/genotype frequency n (\%) } & \multicolumn{3}{|c|}{$\mathrm{P}$ value $[\mathrm{OR}(95 \% \mathrm{CI})]$} \\
\hline & Control & AITD & $\mathrm{GD}$ & HT & AITDs vs. C & GD vs. C & HT vs. C \\
\hline \multicolumn{8}{|l|}{ rs2941522 } \\
\hline GG & $69(8.48)$ & $103(11.26)$ & $75(12.42)$ & $28(9.00)$ & \multirow{2}{*}{$\begin{array}{l}0.054[1.37(0.99-1.89)] \\
\text { reference }\end{array}$} & \multirow{2}{*}{$\begin{array}{l}0.015[1.53(1.08-2.16)] \\
\text { reference }\end{array}$} & \multirow{2}{*}{$\begin{array}{l}0.778[1.07(0.67-1.69)] \\
\text { reference }\end{array}$} \\
\hline $\begin{array}{c}\mathrm{AG}+\mathrm{AA} \\
\mathrm{rs} 907091\end{array}$ & $745(91.52)$ & $812(88.74)$ & $529(87.58)$ & $283(90.99)$ & & & \\
\hline CC & $65(7.98)$ & $108(11.80)$ & $79(13.08)$ & $29(9.33)$ & \multirow{3}{*}{$\begin{array}{c}0.008[1.54(1.12-2.13)] \\
\text { reference }\end{array}$} & \multirow{3}{*}{$\begin{array}{c}0.002[1.73(1.23-2.45)] \\
\text { reference }\end{array}$} & \multirow{3}{*}{$\begin{array}{c}0.468[1.19(0.75-1.87)] \\
\text { reference }\end{array}$} \\
\hline $\mathrm{CT}+\mathrm{TT}$ & $749(92.02)$ & $807(88.19)$ & $525(86.92)$ & $282(90.67)$ & & & \\
\hline rs1453559 & & & & & & & \\
\hline GG & $48(5.89)$ & $84(9.18)$ & $62(10.26)$ & $22(7.07)$ & \multirow{3}{*}{$\begin{array}{c}0.010[1.61(1.12-2.33)] \\
\text { reference }\end{array}$} & \multirow{3}{*}{$\begin{array}{c}0.002[1.86(1.25-2.74)] \\
\text { reference }\end{array}$} & \multirow{3}{*}{$\begin{array}{c}0.465[1.22(0.72-2.05)] \\
\text { reference }\end{array}$} \\
\hline $\mathrm{AG}+\mathrm{AA}$ & $766(94.10)$ & $831(90.82)$ & $542(89.74)$ & $289(92.93)$ & & & \\
\hline rs12150079 & & & & & & & \\
\hline AA & $29(3.56)$ & $52(5.68)$ & $36(5.96)$ & $16(5.15)$ & \multirow{3}{*}{$\begin{array}{c}0.037[1.63(1.03-2.60)] \\
\text { reference }\end{array}$} & \multirow{3}{*}{$\begin{array}{c}0.033[1.72(1.04-2.83)] \\
\text { reference }\end{array}$} & \multirow{3}{*}{$\begin{array}{c}0.226[1.47(0.79-2.74)] \\
\text { reference }\end{array}$} \\
\hline $\mathrm{AG}+\mathrm{GG}$ & $785(96.44)$ & $863(94.32)$ & $568(94.04)$ & $295(94.85)$ & & & \\
\hline rs2872507 & & & & & & & \\
\hline AA & $46(5.65)$ & 82(8.96) & $61(10.10)$ & $21(6.75)$ & \multirow{2}{*}{$\begin{array}{c}0.009[1.64(1.13-2.39)] \\
\text { reference }\end{array}$} & \multirow{2}{*}{$\begin{array}{c}0.002[1.88(1.26-2.79)] \\
\text { reference }\end{array}$} & \multirow{2}{*}{$\begin{array}{c}0.485[1.21(0.71-2.06)] \\
\text { reference }\end{array}$} \\
\hline $\mathrm{AG}+\mathrm{GG}$ & $768(94.35)$ & $833(91.04)$ & $543(89.90)$ & $290(93.25)$ & & & \\
\hline
\end{tabular}


homozygotes in GD group also showed higher frequencies compared with those in the control group $(\mathrm{p}=0.015, \mathrm{OR}=1.53$ for GG in rs2941522; p = 0.002 , OR $=1.73$ for $\mathrm{CC}$ in rs907091; $\mathrm{p}=0.002, \mathrm{OR}=$ 1.86 for GG in rs1453559; $\mathrm{p}=0.033, \mathrm{OR}=1.72$ for $\mathrm{AA}$ in rs12150079; and $\mathrm{p}=0.002, \quad \mathrm{OR}=1.88$ for AA in rs2872507). However, in HT patients group, no locus presented a statistical difference in allele or genotype frequencies compared with controls.

After stratified by gender in GD patients (Table 6), the minor allele frequencies of all the 5 SNPs were much higher in female GDs than in female controls. Similarly, mutant homozygotes (GG in rs2941522; CC in rs907091; GG in rs1453559 and AA in rs2872507) were more frequently detected in the female GD group than those in the female control group. On the contrary, these allelic or genotypic frequencies showed no statistical differences between male GD patients and controls (Table 3, OR was not shown if $p>0.05$ ).

\section{Associations between Genotype and Clinical Phenotype}

In the correlation analysis, we could not find that alleles or genotypes were correlated with a specific clinical phenotype. In GD patients, no significant difference was observed in GD patients with or without ophthalmopathy (Table 7). There were no correlations between genotypes and other clinical features either ( $p>0.05$, data not shown), such as adolescents $(<18$ years old) or adults, with or without hypothyridism in HT group, with or without family history in both GD and HT group.

\section{Functional Predictions}

The functions of the 5 variants were extracted from the website of SNPinfo. Rs1453559 and rs12150079 were located
Table 7. The allelic and genotypic frequencies of 5 SNPs in GD patients with or without ophthalmopathy

\begin{tabular}{|c|c|c|c|c|}
\hline \multirow{2}{*}{ SNP ID } & & \multicolumn{2}{|r|}{ GD } & \multirow{2}{*}{$\begin{array}{c}\text { P value } \\
\text { AITDs vs. C }\end{array}$} \\
\hline & & Ophthalmopathy & Non-Ophthalmopathy & \\
\hline \multirow{5}{*}{ rs2941522 } & G & $76(35.19)$ & $329(33.17)$ & \multirow{3}{*}{0.569} \\
\hline & A & $140(64.81)$ & $663(66.83)$ & \\
\hline & GG & $12(11.11)$ & $63(13.43)$ & \\
\hline & AG & $52(48.14)$ & $203(40.92)$ & \multirow{2}{*}{0.388} \\
\hline & AA & $44(40.74)$ & $230(46.37)$ & \\
\hline \multirow{5}{*}{ rs907091 } & $\mathrm{C}$ & $77(35.65)$ & $333(33.57)$ & \multirow{3}{*}{0.559} \\
\hline & $\mathrm{T}$ & $139(64.35)$ & $659(66.43)$ & \\
\hline & CC & $13(12.03)$ & $66(13.30)$ & \\
\hline & CT & $51(47.22)$ & $201(40.52)$ & \multirow{2}{*}{0.440} \\
\hline & TT & $44(40.74)$ & $229(46.16)$ & \\
\hline \multirow{5}{*}{ rs1453559 } & G & $69(31.94)$ & $300(30.24)$ & \multirow{2}{*}{0.623} \\
\hline & A & $147(68.06)$ & $692(69.76)$ & \\
\hline & GG & $11(10.18)$ & $51(10.28)$ & \multirow{3}{*}{0.777} \\
\hline & AG & $47(43.51)$ & 198(39.91) & \\
\hline & AA & $50(46.29)$ & $247(49.79)$ & \\
\hline \multirow{5}{*}{ rs12150079 } & A & $55(25.46)$ & $228(22.98)$ & \multirow{3}{*}{0.436} \\
\hline & G & $161(74.54)$ & 764(77.02) & \\
\hline & AA & $5(4.63)$ & $31(6.25)$ & \\
\hline & AG & $45(41.66)$ & $166(33.46)$ & \multirow{2}{*}{0.253} \\
\hline & GG & $58(53.70)$ & $299(60.28)$ & \\
\hline \multirow{5}{*}{ rs2872507 } & A & $70(32.41)$ & $298(30.04)$ & \multirow{2}{*}{0.493} \\
\hline & G & 146(67.59) & 694(69.96) & \\
\hline & AA & $10(9.26)$ & $51(10.28)$ & \multirow{3}{*}{0.429} \\
\hline & AG & $50(46.29)$ & $196(39.52)$ & \\
\hline & GG & $48(44.44)$ & $249(50.20)$ & \\
\hline
\end{tabular}

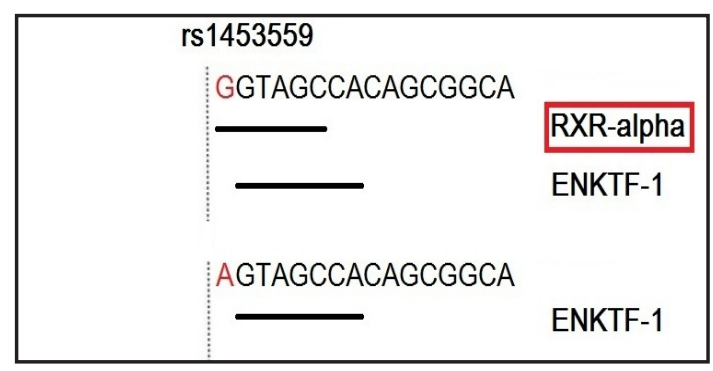

Fig. 1. Changed transcription factor binding sites (TFBS) of rs1453559. G>A mutation was marked by red letter. TFBS were shown below the gene sequence. If mutant A was carried, transcription factor RXR-alpha could not bind to this site. 
at transcription factor binding sites (TFBS). For rs1453559, transcription factor of RXR-alpha could bind on this site when nucleotide $\mathrm{A}$ was located. If mutant $\mathrm{G}$ was carried, this TFBS was predicted not to exist (Fig. 1). As shown in Fig. 2 for rs12150079, if nucleotide $\mathrm{G}$ was changed into $\mathrm{A}$, two new TFBS of GATA-1 and GATA-2 would appear. Rs907091 was a miRNA binding site, which was predicted to be targeted by miRNAs of hsa-miR-326, hsa-miR-330-5p and hsa-miR-450b-3p when nucleotide $T$ was present. However, only hsa-miR-326 could target this locus if nucleotide $\mathrm{T}$ was changed into C (Fig. 3). This indicates that polymorphisms of these sites could change the transcription efficient of this gene.

\section{Discussion}

AITDs may share some common genetic and environmental etiology, such as Th1/ Th2 dysregulations and susceptible genes like CTLA-4. It is more likely to manifest familial aggregation among the patient of GD or HT. SNP is the variation of base sequence in genome. Therefore, a changed genotype could exert an influence on receptor's binding or protein structure, thus affect the efficiency of gene transcription. As a result, SNP could arouse the susceptibility of certain diseases due to the changed biological property of genotype. In our research, we hypothesized that the SNPs within IKZF3 gene could be associated with the occurrence of GD or HT. IKZF3 has been proven to exert a critical role in the development of immunocytes. This protein is composed of 509 amino acids, therefore forms homodimers, or forms heterodimers with the Ikaros. IKZF3 deficient mice will present chronically activated B lymphocytes as well as spontaneous production of autoantibodies [16]. Several isoforms of IKZF3 have been observed in normal or leukaemic B cells [17]. Recent research reported that the protein encoded by IKZF3 gene could induce the expression of immunosuppressive enzymes [18]. Recently, more and more variants of $I K Z F 3$ were successfully identified in a series of autoimmune diseases.

To our knowledge, this is the first research focused on the relationship between IKZF3 polymorphisms and the susceptibility with AITDs. Our results showed that the minor alleles of rs2941522, rs907091, rs1453559, rs12150079 and rs2872507 were statistically associated with an increased risk in GD patients, but not in HT patients. As we known, the feature of GD is hyperthyroidism, which is due to the overproduction of thyroid hormone induced by specific autoantibodies against TSHR, and B cell is a key regulator of GD. As the most common cause for hypothyroidism, HT, on the other hand, is characterized by lymphocytic infiltration and TPOAb/TgAb overproduction. Discrepancies exist in the lymphocytic profiles [19] and the Fc receptors expressed on B cells [20] between the two diseases. HT is primarily a T lymphocyte mediated disease, and whether B cells play a pathogenic role is still unclear [21]. 
Given the essential role of $I K Z F 3$ in the regulation of B cells' differentiation and proliferation, it can be presumed that IKZF3 may be an important element for the pathogenesis of GD rather than HT.

Rs1453559 is located in the 5'UTR, an important area which regulates the transcription efficiency of genes [22]. This polymorphism has been considered as a promising diseaserelated gene of PBC [15] and asthma [12] in Canadian and Italian [15] or the British [12] populations, suggesting that rs1453559 variant might contribute to autoimmune diseases. Taken our results together, it can be proved that rs1453559 A>G was a susceptible factor for GD. Similarly, the variant of rs12150079 has been reported to be associated with other established Type 1 Diabetes (T1D) SNPs in 419 T1D children [23]. Our results showed that the minor A allele and AA genotype were increased in GD, which were consistent with Cai's report that rs12150079 contributed to the etiology of SLE in Chinese Han population [8]. As autoimmune diseases might share some genetic predispositions, the B-cell affecting gene, IKZF3 could lead to the occurrence of GD.

Rs907091 is located in the 3'UTR, a region which can be recognized and combined by certain miRNA or siRNA, thereby influencing the mRNA transcription and translation. The aberrant profile of miRNA expressions was proved to be a risk factor for GD [24], though the role of rs907091 has not been clearly illustrated. Recently, rs907091 was also referred to as an expression quantitative trait locus for IKZF3 in lymphoblastoid cell lines [25]. This SNP has been indicated as a susceptible factor for multiple sclerosis and SLE. It has been observed in Keshari's research that rs907091 was in strong linkage with the multiple sclerosis in Norwegian patients [26]. In consistent with our research, a study confirmed rs907091 was associated with SLE in the Chinese Han population. However, the frequency of C allele and CC genotype appeared to be much lower in the case group [8], which indicated that the mutant genotypes were associated with a lower incidence of disease, in contrast with a higher one in our study. As for rs2872507, a resent GWAS study [27] reported its correlation with Crohn's disease and Type 2 Diabetes (T2D). Another GWAS meta-analysis study of 16, 659 RA patients and 49, 174 controls reported that rs2872507 surpassed genome-wide significance [11]. However, in the previous SLE cohort [8], this variant did not meet statistical significance. These results suggested the heterogeneity of autoimmune disorders and the IKZF3 could play different roles in these diseases.

We investigated the association between genotype and phenotype. The female GD patients are more likely to carry more mutant alleles than female controls. In male GD group, the frequency of mutant alleles is also higher than that in male control group, though it did not show a statistical significance. We also noticed that IKZF3 SNPs showed no difference between patients with Graves' ophthalmopathy (GO) and without GO. A number of studies have proved that the polymorphisms of some genes, such as TSHR and HLA-DR3, could induce GO or thyroid-associated ophthalmopathy (TAO). However, our results suggest IKZF3 may not be a susceptible gene for GO. It is known that GO has some unique pathogenesis compared with GD, nevertheless, IKZF3 is probably not the gene functioning as a divide for GD and GO. Therefore, more researches about IKZF3 genotypes with clinical manifestations are required to reveal the underlying mechanisms.

In the previous publications, the sample size of mutant homozygotes was too small, which might lead to a biased result. Therefore, although the homozygotes analysis has many advantages, the heterozygote analysis could be more reliable. Our study was able to recruit a large population including relatively sufficient homozygote carriers, which avoided the bias caused by small sample size. The statistical power was strong enough (larger than 0.8 ) for assessing the relationship between GD patients and controls. What is more, the sample size of patients and controls was large enough to reduce the type I and II errors.

It is also worthwhile to show the limitations in our study. First of all, the five SNPs of IKZF3 were selected from a linkage disequilibrium block, which provided limited informations, thus cannot fully represent the feature of IKZF3 gene. The other locus of IKZF3 was also reported to be associated with autoimmune diseases. Rs12946510 was reported to be an expressional quantitative trait loci (eQTL) of IKZF3, which increased the susceptibility with inflammatory

\section{KARGER}


bowel diseases [28]. Future studies using gene sequencing analysis could provided further evidences of IKZF3. Besides, the associations between IKZF3 polymorphisms and GD, as well as the functional predictions, cannot prove that these SNPs are the true functional variant of the disease. In addition, the roles of the five SNPs have not been completely understood. Therefore, further functional studies are required to reveal the pathomechanism of IKZF3 polymorphisms.

\section{Conclusion}

Our study firstly reported that the polymorphisms of rs2941522, rs907091, rs1453559, rs12150079 and rs2872507 in IKZF3 are associated with the susceptibility of GD, but not with that of HT. These findings could help to boost the cognition of autoimmune thyroid diseases.

\section{Acknowledgements}

This project is supported by grants from the National Natural Science Foundation of China (No. 81471004 and 81670722) and the Key Disciplines Development of Shanghai Jinshan District (No. JSZK2015A02).

Author Contributions Statement: Ling Li wrote the main manuscript text. Ling Li and Xiao lian Ding screened this gene out of susceptibility genes. Xuan Wang, Qiuming Yao and Xiaoqing Shao identified the variant loci. Xiaofei An, Ni Yan, Yanfei Jiang, Wen Wang, Liangfeng Shi and Qiu Qin accomplished DNA extraction and purification. Ronghua Song helped with calculations using Haploview software. Jin-an Zhang* and Peilong Sun* designed this study. All authors have reviewed the manuscript.

The work has not been published previously.

Ethical Approval: This study received the approval of Institutional Reviewer Board of Jinshan Hospital of Fudan University. All experiments were performed in accordance with relevant guidelines and regulations.

\section{Disclosure Statement}

The authors declared no conflict of interest.

\section{References}

1 C Li, J Yuan, YF Zhu, XJ Yang, Q Wang, J Xu, ST He, JA Zhang: Imbalance of Th17/Treg in Different Subtypes of Autoimmune Thyroid Diseases. Cell Physiol Biochem 2016;40:245-252.

-2 JA Gebe, E Swanson, WW Kwok: HLA class II peptide-binding and autoimmunity. Tissue Antigens 2002;59:78-87.

-3 X Chu, CM Pan, SX Zhao, J Liang, GQ Gao, XM Zhang, GY Yuan, CG Li, LQ Xue, M Shen, W Liu, F Xie, SY Yang, HF Wang, JY Shi, WW Sun, WH Du, CL Zuo, JX Shi, BL Liu, CC Guo, M Zhan, ZH Gu, XN Zhang, F Sun, ZQ Wang, ZY Song, CY Zou, WH Sun, T Guo, HM Cao, JH Ma, B Han, P Li, H Jiang, QH Huang, L Liang, LB Liu, G Chen, Q Su, YD Peng, JJ Zhao, G Ning, Z Chen, JL Chen, SJ Chen, W Huang, HD Song, D China Consortium for Genetics of Autoimmune Thyroid: A genome-wide association study identifies two new risk loci for Graves' disease. Nat Genet 2011;43:897-901.

4 K Georgopoulos, S Winandy, N Avitahl: The role of the Ikaros gene in lymphocyte development and homeostasis. Annu Rev Immunol 1997;15:155-176.

5 S Ma, S Pathak, M Mandal, L Trinh, MR Clark, R Lu: Ikaros and Aiolos inhibit pre-B-cell proliferation by directly suppressing c-Myc expression. Mol Cell Biol 2010;30:4149-4158. 


\section{Cellular Physiology Cell Physiol Biochem 2018;45:1787-1796 \begin{tabular}{ll|l} 
and Biochemistry & $\begin{array}{l}\text { DOI: 10.1159/000487870 } \\
\text { Published online: March 05, } 2018\end{array}$ & $\begin{array}{l}\text { (c) } 2018 \text { The Author(s). Published by S. Karger AG, Basel } \\
\text { www.karger.com/cpb }\end{array}$
\end{tabular}}

Li et al.: IKZF3 Polymorphisms with GD

6 S Ma, S Pathak, L Trinh, R Lu: Interferon regulatory factors 4 and 8 induce the expression of Ikaros and Aiolos to down-regulate pre-B-cell receptor and promote cell-cycle withdrawal in pre-B-cell development. Blood 2008;111:1396-1403.

7 JH Wang, N Avitahl, A Cariappa, C Friedrich, T Ikeda, A Renold, K Andrikopoulos, L Liang, S Pillai, BA Morgan, K Georgopoulos: Aiolos regulates B cell activation and maturation to effector state. Immunity 1998;9:543-553.

8 X Cai, Y Qiao, C Diao, X Xu, Y Chen, S Du, X Liu, N Liu, S Yu, D Chen, Y Jiang: Association between polymorphisms of the IKZF3 gene and systemic lupus erythematosus in a Chinese Han population. PLoS One 2014; 9:e108661.

-9 CJ Lessard, I Adrianto, JA Ice, GB Wiley, JA Kelly, SB Glenn, AJ Adler, H Li, A Rasmussen, AH Williams, J Ziegler, ME Comeau, M Marion, BE Wakeland, C Liang, PS Ramos, KM Grundahl, CJ Gallant, ME AlarconRiquelme, GS Alarcon, et a.: Identification of IRF8, TMEM39A, and IKZF3-ZPBP2 as susceptibility loci for systemic lupus erythematosus in a large-scale multiracial replication study. Am J Hum Genet 2012;90:648660.

10 J Sun, G Matthias, MJ Mihatsch, K Georgopoulos, P Matthias: Lack of the transcriptional coactivator OBF1 prevents the development of systemic lupus erythematosus-like phenotypes in Aiolos mutant mice. J Immunol 2003;170:1699-1706.

11 FA Kurreeman, EA Stahl, Y Okada, K Liao, D Diogo, S Raychaudhuri, J Freudenberg, Y Kochi, NA Patsopoulos, N Gupta, C investigators, C Sandor, SY Bang, HS Lee, L Padyukov, A Suzuki, K Siminovitch, J Worthington, PK Gregersen, LB Hughes, RJ Reynolds, SL Bridges, Jr., SC Bae, K Yamamoto, RM Plenge: Use of a multiethnic approach to identify rheumatoid- arthritis-susceptibility loci, 1p36 and 17q12. Am J Hum Genet 2012;90:524-532.

12 S Marinho, A Custovic, P Marsden, JA Smith, A Simpson: 17q12-21 variants are associated with asthma and interact with active smoking in an adult population from the United Kingdom. Ann Allergy Asthma Immunol 2012;108:402-411 e409.

13 N Acevedo, LE Reinius, D Greco, A Gref, C Orsmark-Pietras, H Persson, G Pershagen, G Hedlin, E Melen, A Scheynius, J Kere, C Soderhall: Risk of childhood asthma is associated with CpG-site polymorphisms, regional DNA methylation and mRNA levels at the GSDMB/ORMDL3 locus. Hum Mol Genet 2015;24:875890.

14 M Blekic, B Kljaic Bukvic, N Aberle, S Marinho, J Hankinson, A Custovic, A Simpson: 17q12-21 and asthma: interactions with early-life environmental exposures. Ann Allergy Asthma Immunol 2013;110:347-353 e342.

15 BD Juran, GM Hirschfield, P Invernizzi, EJ Atkinson, Y Li, G Xie, R Kosoy, M Ransom, Y Sun, I Bianchi, EM Schlicht, A Lleo, C Coltescu, F Bernuzzi, M Podda, C Lammert, R Shigeta, LL Chan, T Balschun, M Marconi, et al.: Immunochip analyses identify a novel risk locus for primary biliary cirrhosis at 13q14, multiple independent associations at four established risk loci and epistasis between 1p31 and 7q32 risk variants. Hum Mol Genet 2012;21:5209-5221.

-16 C Schmitt, C Tonnelle, A Dalloul, C Chabannon, P Debre, A Rebollo: Aiolos and Ikaros: regulators of lymphocyte development, homeostasis and lymphoproliferation. Apoptosis 2002;7:277-284.

-17 J Liippo, KP Nera, E Veistinen, A Lahdesmaki, V Postila, E Kimby, P Riikonen, L Hammarstrom, J Pelkonen, 0 Lassila: Both normal and leukemic B lymphocytes express multiple isoforms of the human Aiolos gene. Eur J Immunol 2001;31:3469-3474.

18 CM Scarlata, C Celse, P Pignon, M Ayyoub, D Valmori: Differential expression of the immunosuppressive enzyme IL4I1 in human induced Aiolos+, but not natural Helios+, FOXP3+ Treg cells. Eur J Immunol 2015;45:474-479.

19 AM Ramos-Levi, M Marazuela: Pathogenesis of thyroid autoimmune disease: the role of cellular mechanisms. Endocrinol Nutr 2016;63:421-429.

20 D Rostamzadeh, MH Dabbaghmanesh, M Shabani, A Hosseini, Z Amirghofran: Expression Profile of Human Fc Receptor-Like 1, 2, and 4 Molecules in Peripheral Blood Mononuclear Cells of Patients with Hashimoto's Thyroiditis and Graves' Disease. Horm Metab Res 2015;47:693-698.

21 B Kristensen: Regulatory B and T cell responses in patients with autoimmune thyroid disease and healthy controls. Dan Med J 2016;63:pii:B5177.

-22 M Thomas, MA Sukhai, S Kamel-Reid: An emerging role for retinoid X receptor alpha in malignant hematopoiesis. Leuk Res 2012;36:1075-1081. 


\section{Cellular Physiology Cell Physiol Biochem 2018;45:1787-1796 \begin{tabular}{ll|l} 
DOI: 10.1159/000487870 & $\begin{array}{l}\text { O 2018 The Author(s). Published by S. Karger AG, Basel } \\
\text { www.karger.com/cpb }\end{array}$ \\
\hline
\end{tabular} \\ Li et al.: IKZF3 Polymorphisms with GD}

23 E Witso, $\mathrm{O}$ Cinek, G Tapia, CA Brorsson, LC Stene, HK Gjessing, T Rasmussen, R Bergholdt, FM Pociot, KS Ronningen: Genetic Determinants of Enterovirus Infections: Polymorphisms in Type 1 Diabetes and Innate Immune Genes in the MIDIA Study. Viral Immunol 2015;28:556-563.

24 Q Qin, X Wang, N Yan, RH Song, TT Cai, W Zhang, LJ Guan, FS Muhali, JA Zhang: Aberrant expression of miRNA and mRNAs in lesioned tissues of Graves' disease. Cell Physiol Biochem 2015;35:1934-1942.

-25 T Lappalainen, M Sammeth, MR Friedlander, PA t Hoen, J Monlong, MA Rivas, M Gonzalez-Porta, N Kurbatova, T Griebel, PG Ferreira, M Barann, T Wieland, L Greger, M van Iterson, J Almlof, P Ribeca, I Pulyakhina, D Esser, T Giger, A Tikhonov, et al.: Transcriptome and genome sequencing uncovers functional variation in humans. Nature 2013;501:506-511.

-26 PK Keshari, HF Harbo, KM Myhr, JH Aarseth, SD Bos, T Berge: Allelic imbalance of multiple sclerosis susceptibility genes IKZF3 and IQGAP1 in human peripheral blood. BMC Genet 2016;17:59.

27 A Lacour, D Ellinghaus, S Schreiber, A Franke, T Becker: Haplotype synthesis analysis reveals functional variants underlying known genome-wide associated susceptibility loci. Bioinformatics 2016;32:21362142.

-28 AF Di Narzo, LA Peters, C Argmann, A Stojmirovic, J Perrigoue, K Li, S Telesco, B Kidd, J Walker, J Dudley, J Cho, EE Schadt, A Kasarskis, M Curran, R Dobrin, K Hao: Blood and Intestine eQTLs from an AntiTNF-Resistant Crohn's Disease Cohort Inform IBD Genetic Association Loci. Clin Transl Gastroenterol 2016;7:e177. 